\section{Intubation: dogs, cats and rabbits}

\section{Vicky Ford-Fennah}

Intubation is one of the most commonly performed procedures in veterinary medicine, however some patients/patient groups present challenges, complicating this procedure. Intubation is performed to:

- Ensure a patent airway

- Prevent soft tissue obstruction

- Prevent secretions/material obstruction or aspiration

- Provide supplemental oxygen

- Deliver gaseous anaesthesia with minimal environmental contamination

- Allow intermittent positive-pressure ventilation

- Prevent gastric inflation.

A key species difference is tracheal anatomy. Both the cat and rabbit have complete tracheal rings compared to the incomplete rings of the dog. This increases the risk of tracheal damage. They both also have sensitive larynxes, especially the cat (where intubation should not be attempted without application of topical local anaesthetic). This sensitivity increases the associated risk of intubation due to laryngeal spasm, a life-threating complication.

Patient size and conformation significantly impact on difficulty of intubation. Most techniques rely on direct visualization. This can be compromised in patients with:

- Excessive upper airway soft tissues,

for example, brachycephalics

- Difficulty in opening the oral cavity

- Narrow tracheas, for example, rabbits

(comparatively small tracheas compared to body size), brachycephalics

- Normal anatomy - rabbits have long, narrow mouths with large tongue base and large incisors

- Predisposition of regurgitation, for example, non-fasted, pregnant, gastrointestinal disease, brachycephalics.

These patients require additional planning and consideration

Preparation is the key to successful intubation, prevention of complications and management of any issues if they arise. The airway devices that are available include:

- Endotracheal tubes (ETT) - options include cuffed (high-volume low pressure or low volume high pressure), uncuffed, material type (PVC, silicon or red rubber), length (nares to thoracic inlet)

- Human supraglottic airway devices - for example, laryngeal mask airways (LMAs)

- Veterinary-specific supraglottic airway device - V-gels ${ }^{\circledR}$.

All devices have associated advantages and disadvantages. Airway devices should be checked for defects before use.

Intubation should only be attempted once adequate anaesthetic depth is achieved, insufficient depth increases the risk of complications. Patient positioning is also often underestimated. In most instances sternal recumbency is optimal. In both cats and dogs the effective use of a laryngoscope is advantageous.

If difficulties/complications do occur, strategies that can be used include the use of:

- Dog urinary catheter $(3.5 \mathrm{~mm}$ ETT connector allows attachment to breathing system, allowing oxygen supplementation), stylets or bougies can be used to 'rail road' an ETT

- Endoscope - visualization

- Tracheostomy tubes

- Pharyngotomy.

Capnography, pulse oximetry and electrocardiogram (ECG) are all useful monitoring aids during difficult airway management.

Correct airway device positioning can be confirmed with:

- Capnography (gold standard)

- Tube fogging

- Direct visualization

- Concurrent observation of thoracic and reservoir bag movement

- Thoracic auscultation.

All airway devices should be secured effectively to prevent loss of patency or tracheal damage due to movement.

\section{KEY LEARNING OBJECTIVES}

- Have an understanding of the anatomical differences between the dog, cat and rabbit airway

- Understand the rationale behind the use of different airway-management techniques and how to use these safely

- Understand techniques that can be used in a difficult airway situation

\section{MULTIPLE CHOICE QUESTIONS}

1. What is the 'gold standard' method to confirm correct positioning of an airway device?
(A) Use a hair pluck held in front of device to check for air movement
(B) Compress chest
(C) Capnography
(D) Feel for air movement

2. What size ETT connector can be inserted in to the luer connector of a dog urinary catheter to connect it to a breathing system to allow oxygen supplementation?
(A) $3.5 \mathrm{~mm}$
(B) $2 \mathrm{~mm}$
(C) $5 \mathrm{~mm}$
(D) $4 \mathrm{~mm}$

3. What equipment can be used to aid placement of an ETT in a difficult airway situation?
(A) Laryngoscope
(B) Stylet
(C) Dog urinary catheter
(D) All of the above 